\title{
Aktarımlı Öğrenme ile SENTINEL-2 Görüntülerinden Kıyı Çizgisi Bölütlemesi
}

\author{
Selennur Karagöl*1 ${ }^{\circledR}$, Bülent Bayram² ${ }^{\circledR}$, Fırat Erdem³ ${ }^{\circledR}$, Tolga Bakırman ${ }^{\circledR}$ \\ ${ }_{1}^{1}$ ıldız Teknik Üniversitesi, Fen Bilimleri Enstitüsü, Uzaktan Algılama ve CBS Anabilim Dalı, İstanbul, Türkiye \\ ${ }^{2}$ Yıldız Teknik Üniversitesi, İnşaat Fakültesi, Harita Mühendisliği, İstanbul, Türkiye \\ ${ }^{3}$ Eskișehir, Teknik Üniversitesi, Yer ve Uzay Bilimleri Enstitüsü, Eskișehir, Türkiye \\ 4İstanbul Teknik Üniversitesi, Uydu Haberleşmesi ve Uzaktan Algılama Araștırma ve Uygulama Merkezi, İstanbul, Türkiye
}

\begin{abstract}
Anahtar Kelimeler: Derin Öğrenme Aktarımlı Öğrenme Kıyı Çizgisi Çıkartma SENTINEL-2

LANDSAT-8
\end{abstract}

\begin{abstract}
ÖZ
Kıyı çizgileri küresel ısınma, nüfus artışı, çevre kirliliği, kentleşme etkileriyle sürekli değișir. Doğal ve antropojenik etkilerle meydana gelen değişikleri tespit etmek için kıyı alanlarının izlenmesi gerekmektedir. Kıyı alanlarındaki değișimlerin sürdürülebilir bir şekilde izlenmesi, kıyı kaynak yönetimi, çevresel koruma ve planlama açısından oldukça önemli rol oynamaktadır. Uydu görüntüleri bu amaç için doğru, güvenilir, zamansal ve güncel bilgiler sağlamaktadır. Derin öğrenme(DL) ve aktarımlı öğrenme(TL) yaklaşımları kıyı çizgisi çıkartılmasında yeni olanaklar sağlamaktadır. Sunulan çalıșmada, SENTINEL-2 görüntülerinden aktarımlı öğrenmeye dayalı, U-NET mimarisi kullanılarak, bir kara ve su bölütlemesi yaklaşımı önerilmiştir. Önceden eğitilmiş modele ait özellikler ve ağırlıklar için, LANDSAT-8 görüntüleri ile gerçekleştirilen derin öğrenme çalıșmasından yararlanılmıștır. U-Net mimarisi kullanılan ağda, mavi, kırmızı ve yakın kızıl ötesi bantlarından oluşan tam çerçeve SENTINEL-2 görüntülerinden 8'i eğitim, 7'si test aşamasında kullanılmıştır. Tam çerçeve görüntüler 512x512 boyutlarında kırpılarak eğitim ve test için sırasıyla 115 ve 235 görüntü parçası oluşturulmuştur. Ortalama doğruluk, duyarlılık, hassasiyet, özgünlük ve F-skor değerleri sırasıyla 0.9917, 0.9927, 0.9908, 0.9907 ve 0.9917 olarak hesaplanmıştır. Çalışmanın sonuçlarına göre, aktarımlı öğrenme kullanılarak az miktarda görüntü ile yüksek doğruluklu kıyı çizgisi elde etmek mümkündür.
\end{abstract}

\section{Shoreline Segmentation from SENTINEL-2 Imagery by Transfer Learning}

\author{
Keywords: \\ Deep Learning \\ Transfer Learning \\ Shoreline Extraction \\ SENTINEL-2 \\ LANDSAT-8
}

\begin{abstract}
Global warming, increasing population, environmental pollution and urbanization can constantly affect coastal areas. Therefore, sustainable monitoring of coastal zones is vital to detect changes which can occur due to natural and anthropogenic effects. Thus, sustainable shoreline monitoring is essential for coastal resource management, environmental protection and planning. Satellite images provide accurate, reliable, temporal and up-to-date information for this purpose. State-of-the-art deep learning (DL) and transfer learning approaches brought new opportunities for shoreline extraction. In this study, a transfer learning based water-body segmentation framework with U-Net architecture from SENTINEL-2 imagery has been proposed. The pre-trained weights have been obtained from another study which is a network trained with LANDSAT-8 imageries. The training of used U-Net architecture was carried out using SENTINEL-2 imagery which consists of blue, red and NIR bands with 8 and 7 full frames for training and testing, respectively. Images have been cropped as $512 \times 512$ pixels and 115 and 235 patches have been created for the training and testing dataset, respectively. Average accuracy, recall, precision, specivity and F-score of the model values has been calculated as $0.9917,0.9927,0.9908,0.9907$ and 0.9917 , respectively. The results show that it is possible to obtain shoreline with high accuracy with limited data using transfer learning.
\end{abstract}




\section{GİRIŞ}

Kıyı alanları, insan etkileri ve doğal sebeplerden kaynaklanan dinamik değişiklerin olduğu, ekonomik ve sosyal açıdan nitelikli bölgelerdir. Mineral kaynakları, petrol-gaz kaynakları, gelgit-dalga enerji kaynakları ve diğer yenilenebilir enerji kaynakları bakımından yerleşimler kıy alanlarında yoğunlaşmaktadır (Zhang vd., 2013). Kıyı bölgelerinde artan nüfusla birlikte, kıyı değişiminin incelenmesi araştırmacıların en önem verdiği konulardan biridir (Moore, 2000). Kıyı çizgisinde meydana gelen değișimlerin hızlı ve doğru bir şekilde belirlenmesi sadece kıyı ıslahı, kentsel büyüme ve liman geliştirme faaliyetleri için değil aynı zamanda denizcilik ekonomisi ve denizcilik araştırmaları için de önemli bir konudur (Zhang vd., 2013).

Kıyı çizgisini çıkartmak için fotogrametrik yöntemler, GPS teknolojisi ve yersel ölçüler, uzaktan algılama gibi çeşitli yöntemler kullanılmaktadır (Zhang vd., 2013). LANDSAT uydusunun 1972'de kullanılmaya başlanılmasından bu yana optik uzaktan algılama verileri diğer yöntemlerden elde edilen verilere bir alternatif haline gelmiştir (Gens, 2010). Kontrolsüz siniflandırma teknikleri (ISODATA-Iterative Self Organized Data Analysis) bant oranlama, normalize edilmiş fark su indeksi (NDWI) eşik değer ve morfolojik filtreleme, Wavelet dönüșümü, aktif kontur modelleri (Zhang vd., 2013), nesne tabanlı, genetik algoritma, kontrollü ve kontrolsüz sınıflandırma, parçacık sürü optimizayonu (PSO), Mean-shift bölütleme (İncekara vd., 2018) optik uydu görüntülerinden kıyı çizgisi çıkarmak için kullanılan yöntemlere örnek olarak verilebilir. Son yıllarda makine öğrenmesi yöntemleri uzaktan algılama problemlerinde yaygın olarak uygulanmaktadır (Lary vd., 2016). Çeşitli çalışmalarda kıyı çizgisi çıkarımında makine öğrenmesi yöntemleri kullanılmıştır (Dixon \& Candade, 2008; Kalkan vd., 2013; Bayram vd., 2017). Choung \& Jo, 2017 Worldview-2 uydu görüntüleriyle bir makine öğrenmesi yöntemi olan Destek Vektör Makineleri (Support Vector Machines) ile adaptif eşikleme ve NDWI yöntemin kıyı çizgisi çıkarımındaki performansını araştırmıştır.

Derin öğrenme (DL), Goodfellow vd., 2016 tarafından bilgisayarların dünyayı kavram hiyerarşileri açısından anlamasını ve insanlara benzer şekilde karar vermesini sağlayan makine öğrenmesi olarak tanımlanmaktadır. Klasik makine öğrenmesinde özellik çıkartma işlemi kullanıcı tarafından yapılmaktadır ve bu zaman alıcı ve kullanıcı odaklı bir süreçtir. Derin öğrenme yaklaşımında ise özellik çıkartma işlemi otomatik olarak yapılmaktadır (Patterson \& Gibson, 2017).

DL yöntemleri, son yıllarda kıyı alanlarına yönelik çalışmalarda yaygın olarak kullanılmaya başlanmıştır. Yang vd., 2015 LANDSAT uydu görüntülerinde SSAE (Stacked Sparse Autoencoder) derin öğrenme mimarisini kullanarak kara-su bölütlemesi gerçekleștirmişlerdir. Yu vd., 2017 LANDSAT-7 görüntülerinden kıyı çizgilerini çıkarmak için evrişimsel sinir ağları ve lojistik regresyon sınıflandırıcısından oluşan karma bir makine öğrenimi sistemi sunmuştur. Işıkdoğan vd., 2017 DeepWaterMap adlı kodlayıcı-kod çözücü derin öğrenme mimarisine dayalı Tam Konvolüsyonlu Ağ (FCNN) yapısı kullanarak LANDSAT-7 uydu görüntülerinde kara-su bölütlemesi gerçekleştirmişlerdir. Li vd., 2018 kıyı çizgisi bölütlemesi için DeepUNet adlı genişleyen konvolüsyonel sinir ağları tabanlı bir yaklaşım önermiştir. Chen vd., 2018 süper piksel ve konvolüsyonel sinir ağı algoritmalarını kullanarak yüksek çözünürlüklü çok bantlı görüntülerden kıyı çizgisi çlkarmıștır. Song vd., 2020 Worldview-3 ve GeoFen-2 görüntülerinden Mask R-CNN yöntemini kullanarak su sınıfını yüksek doğruluklar ile üretmişlerdir. Erdem vd., 2020 tarafından yapılan çalışmada LANDSAT-8 görüntülerinden su sınıfının çıkarılması için WaterNet adında 5 farklı U-Net derin öğrenme modelinin kombinasyonu kullanan bir yöntem geliştirilmiştir.

Uzaktan Algılama çalışmalarında derin öğrenme modellerinin uygulanmasında aktarımlı öğrenme (transfer learning) yaygın olarak kullanılan bir yöntemdir. Aktarımlı öğrenme ile bir veri seti için yapılan eğitim işlemi sonucunda oluşturulan ağırlık parametresi, bir bașka veri setinin eğitilmesi için bașlangıç ağırlıkları olarak kullanılır. Bu durum, az sayıda veri ile yüksek doğrulukta sonuçlar üretmek açısından avantajlar sağlamaktadır (Torrey \& Shavlik, 2009).

Yapılan literatür araştırmasında SENTINEL-2 görüntülerinden aktarımlı öğrenme yöntemi kullanılarak kara-deniz bölütlemesi ve kıyı çizgisi çıkartmaya yönelik bir çalışmaya rastlanmamıştır. Bu çalıșmada, önceden LANDSAT-8 uydu görüntüleri ile eğitilmiș U-Net mimarisi (Erdem, vd., 2020) ve az sayıda SENTINEL-2 uydu görüntüsü veri seti kullanılarak aktarımlı öğrenme ile kıyı çizgisi çıkarılmıştır.

\section{YÖNTEM}

$\mathrm{Bu}$ çalışmada SENTINEL-2 veri setinden karadeniz bölütlerinin U-Net mimarisi ile elde edilmesi için YTU-WaterNet açık veri seti (URL-1) ile eğitilen U-Net mimarisinin ağırlıkları (Erdem, vd., 2020) başlangıç ağırlıkları olarak kullanılmıştır.

\subsection{Veri}

Sunulan çalışmada, aktarımlı öğrenme için farklı kıyı bölgelerinden alınan 10m konumsal çözünürlüğe ve 15-bit radyometrik çözünürlüğe ve mavi, kırmızı ve yakın kızılötesi bant kombinasyonuna sahip toplam 15 adet SENTINEL-2 uydu görüntüsü kullanılarak eğitim ve test veri 
setleri üretilmiştir. Tablo 1'de SENTINEL-2 uydu görüntülerinin teknik özellikleri gösterilmektedir. Eğitim için 8, test için 7 adet görüntü kullanılmıștır. Eğitim ve test amacıyla kullanılan görüntülerin konumları Şekil 1'de gösterilmektedir.

Tablo 1. SENTINEL-2 uydu görüntüsünün özellikleri (URL-2)

\begin{tabular}{lll}
\hline Bantlar & \multicolumn{1}{c}{ Özellikleri } & $\begin{array}{l}\text { Merkez Dalga } \\
\text { Boyu (nm) }\end{array}$ \\
\hline Bant1 & 60m Kıyı aerosolü & 443 \\
Bant2 & 10m Mavi & 490 \\
Bant3 & 10m Yeşil & 560 \\
Bant4 & 10m Kırmızı & 665 \\
Bant5 & 20m Bitki örtüsü & 705 \\
Bant6 & 20m Bitki örtüsü & 740 \\
Bant7 & 20m Bitki örtüsü & 783 \\
Bant8 & 10m Yakın Kızlötesi & 842 \\
Bant8A & 20m Bitki örtüsü & 865 \\
Bant9 & 60m Su buharı & 945 \\
Bant10 & 60m SWIR & 1375 \\
Bant11 & 20m SWIR & 1610 \\
Bant12 & 20m SWIR & 2190 \\
\hline
\end{tabular}

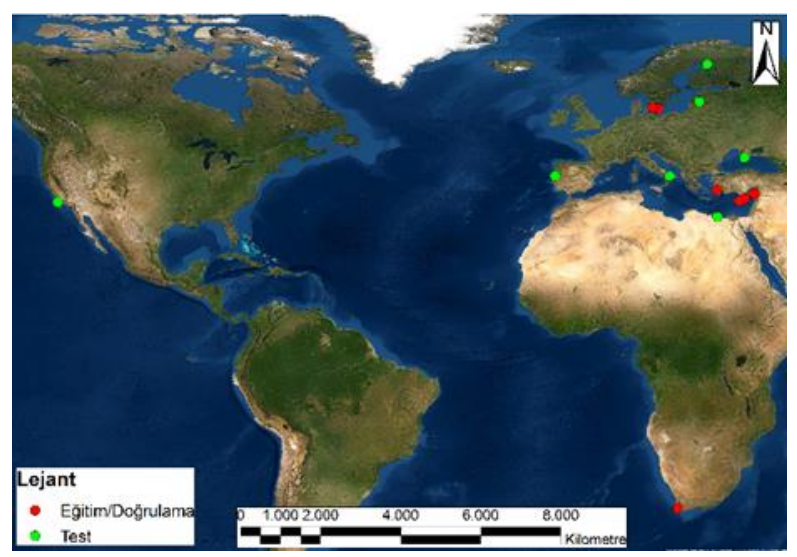

Şekil 1. Eğitim ve test için kullanılan görüntülerin dağılımları

\subsection{Veri Ön İşleme}

Sunulan çalışmada 10980x10980 piksel boyutlarındaki SENTINEL-2 görüntüleri $512 \times 512$ piksel boyutlarında alt görüntülere bölünmüştür ve toplam 350 alt görüntüden oluşan veri seti oluşturulmuştur. Bunlardan 115'i eğitim, 235 adedi test için kullanılmıştır. Etiketli veri setinin üretiminde OpenStreetMap (OSM) (URL-3) kullanılmış, ardından elle düzeltme yapılarak su (siyah) ve kara (beyaz) sınıflarını temsil eden ikili görüntülerden oluşan veri seti hazırlanmıştır. Şekil 2'de örnek görüntüler ve etiketleri sunulmuştur.
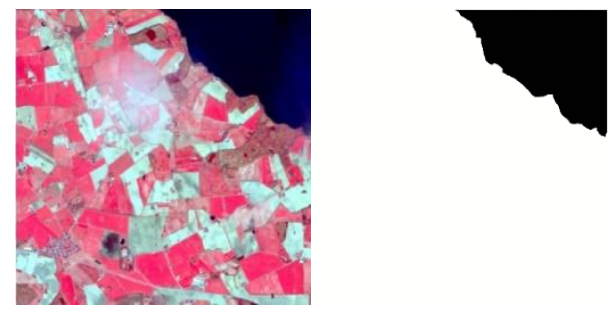

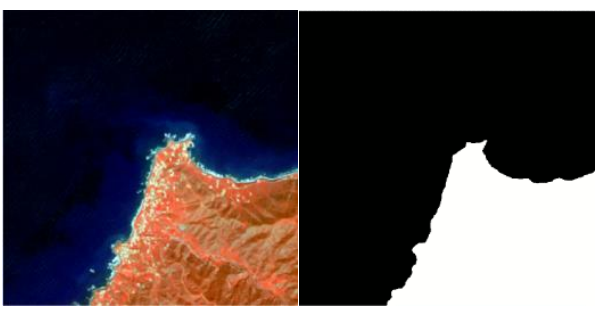

Şekil 2. Örnek görüntüler ve etiketleri

\subsection{Aktarımlı Öğrenme ve U-Net}

Aktarımlı öğrenmede, çoğunlukla önceden eğitilmiş bir modelden elde edilen ağırlık ve özellikler ya doğrudan ya da probleme bağlı olarak eğitilecek ağda öncül veri olarak kullanılır.

U-Net mimarisi, biyomedikal görüntülerin bölütlenmesi için geliştirilen, tam konvolüsyonel ağ (fully convolutional network) temelli bir derin öğrenme mimarisidir. U-Net mimarisi ile az sayıda eğitim verisi kullanılarak başarılı sonuçlar elde edilebilmektedir. (Ronneberger, vd., 2015).

U-Net mimarisi kodlayıcı (contracting path) ve kod çözücü (expansive path) olmak üzere iki bölümden oluşmaktadır. Kodlayıcı kısmında her bir ölçek için, iki adet 3 x 3 konvolüsyon katmanı, ReLU aktivasyon fonksiyonu, $2 \times 2$ maksimum havuzlama operasyonları uygulanmaktadır. Kod çözücü bölümünde ise; yukarı örnekleme, kopyalama (concatenete) ve konvolüsyon katmanları yer almaktadır. Şekil 3'te U-Net mimarisinin genel yapısı gösterilmektedir.

Sunulan çalışmada aktarımlı öğrenme ve U-Net mimarisinde kullanılan parametreler Tablo 2'de gösterilmiștir. Eğitim parametreleri, kullanılan donanımın özellikleri ve literatürdeki çalışmalar dikkate alınarak belirlenmiştir.

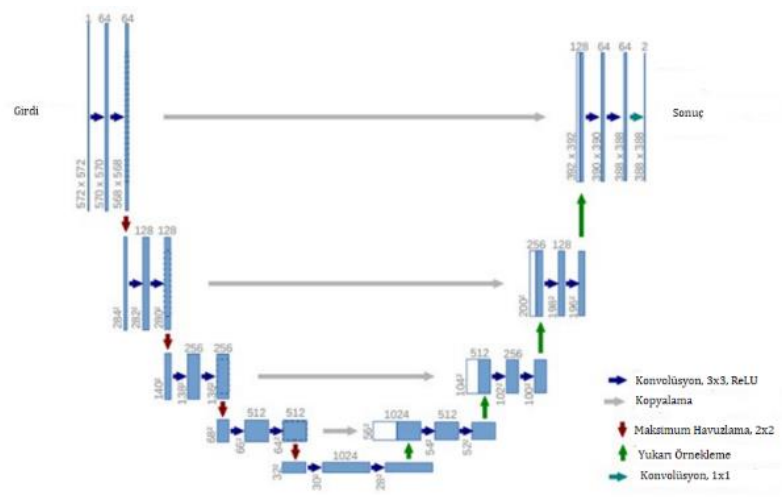

Şekil 3. U-net mimarisi (Ronneberger, vd., 2015)

Tablo 2. Eğitim parametreleri

\begin{tabular}{lcc}
\hline & $\begin{array}{c}\text { Aktarımlı Öğrenme } \\
\text { (Erdem, vd., 2020) }\end{array}$ & $\begin{array}{c}\text { Sunulan Çalışma U- } \\
\text { Net }\end{array}$ \\
\hline $\begin{array}{l}\text { Eğitim } \\
\text { görüntü }\end{array}$ & & \\
sayısı & & \\
$(512 \times 512)$ & 824 & 115 \\
& &
\end{tabular}




\begin{tabular}{|c|c|c|}
\hline $\begin{array}{l}\text { Test } \\
\text { görüntü } \\
\text { sayısı } \\
(512 \times 512)\end{array}$ & 92 & 235 \\
\hline Epok sayısı & 100 & 50 \\
\hline $\begin{array}{l}\text { Loss } \\
\text { Fonksiyonu }\end{array}$ & $\begin{array}{c}\text { Binary } \\
\text { Cross-entropy }\end{array}$ & $\begin{array}{c}\text { Categorical } \\
\text { Cross entropy }\end{array}$ \\
\hline $\begin{array}{l}\text { Aktivasyon } \\
\text { Fonksiyonu }\end{array}$ & ReLU & ReLU \\
\hline $\begin{array}{l}\text { Optimizasy } \\
\text { on }\end{array}$ & Adam & Adadelta \\
\hline $\begin{array}{l}\text { Öğrenme } \\
\text { Oranı }\end{array}$ & 0.0002 & 0.05 \\
\hline $\begin{array}{l}\text { Batch } \\
\text { Boyutu }\end{array}$ & 1 & 2 \\
\hline $\begin{array}{l}\text { Doğruluk } \\
\text { metriği }\end{array}$ & Dice Coefficient & Dice Coefficient \\
\hline
\end{tabular}

\subsection{Doğruluk Ölçütü}

Deniz-kara bölütlemesinin doğruluk değerlendirmesi; doğruluk, duyarlılık, hassasiyet, özgünlük ve F1 metrikleri kullanılarak gerçekleştirilmiştir. Hassasiyet; negatif tahminlerin doğruluğunu, duyarlılık; pozitif tahminlerin doğruluğu, özgünlük; pozitif tahmin doğruluğunu, FSkor ise duyarlılık ve hassasiyet ölçütlerinin harmonik ortalamasıdır. DP; olumlu ve doğru tahmin edilen, DN; olumsuz ve doğru tahmin edilen, YP; olumlu ve yanlış tahmin edilen, YN; olumsuz ve yanlış tahmin edilen olmak üzere doğruluk metrikleri Eşitlik 1' de yer almaktadır.

$$
\begin{aligned}
& \text { Doğruluk }=\frac{D P+D N}{\mathrm{DP}+\mathrm{DN}+\mathrm{YP}+\mathrm{YN}} \\
& \text { Duyarlılık }=\frac{D P}{D P+Y N} \\
& \text { Hassasiyet }=\frac{\mathrm{DP}}{\mathrm{DP}+\mathrm{YP}} \\
& \text { Özgünlük }=\frac{\mathrm{DN}}{D N+Y P} \\
& F-\text { Skor }=\frac{2 * D P}{(2 * D P+Y P+Y N)}
\end{aligned}
$$

\section{BULGULAR}

Sunulan çalışmada, U-Net mimarisi Python v3.7.0 programlama dili, Tensorflow v1.5.0 (URL-4) ve Keras v2.2.4 (URL-5) kütüphaneleri kullanılarak uygulanmıștır. Aktarımlı öğrenmede eğitim ișlemini gerçekleştirmek için SENTINEL-2 uydusuna ait toplam 115 adet alt görüntü kullanılmıştır. Eğitim aşamasında Adadelta optimizasyon algoritması, 0.05 öğrenme oranı ile kullanılmıştır. Epok sayısı 50 olarak belirlenmiştir. SENTINEL-2 görüntülerini eğitmek için başlangıç ağırlıkları olarak önceden eğitilmiş U-Net ağırlıkları (Erdem, vd., 2020) kullanılmıștır. Aktarımlı öğrenmenin eğitimi boyunca elde edilen doğruluklar Şekil 5'te verilmiştir.
Gerçekleştirilen tüm işlemler 11 GB RAM'e sahip NVIDIA GeForce GTX1080 Ti ekran kartı kullanarak yapılmıştır.

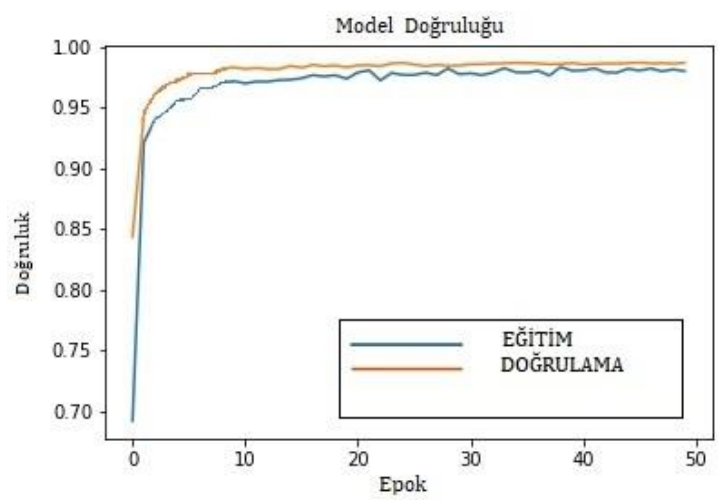

Şekil 5. Aktarımlı öğrenmenin eğitimi boyunca elde edilen doğruluklar

Test görüntülerinden tam SENTINEL-2 çerçevesi (10980x10980) için elde edilen kara-deniz bölütlemesine ilişkin örnek sonuç Şekil 6'da sunulmuştur. Şekil 6'da solda mavi, kırmızı ve yakın kızll ötesi bant kombinasyonunda test görüntüsü ve sağda bölütlenmiş görüntü verilmiştir.
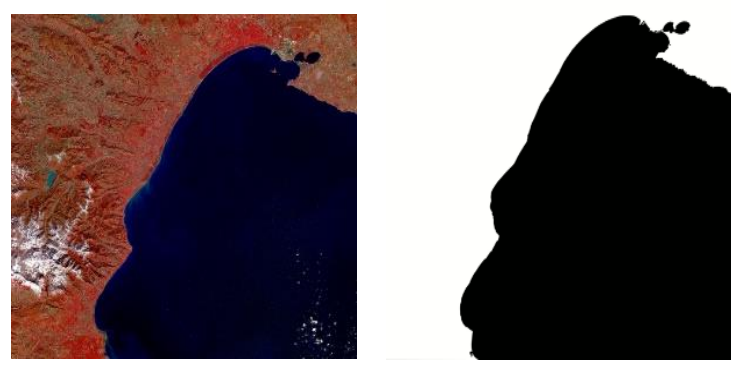

Şekil 6. Test görüntüsü için üretilen ikili görüntü

İkili kodlanmış kara-deniz bölütlerine rastervektör dönüşümü uygulanarak kıyı çizgileri vektör formatta elde edilmiştir. Örnek sonuç Şekil 7' de verilmiştir. Bir tam çerçeve test görüntüsünden $512 \times 512$ alt görüntülerin üretilmesi, bölütleme ve vektör formatta kıyı çizgisinin elde edilmesi sırasıyla $1,1 / 2$ ve 2 dakika sürmektedir.

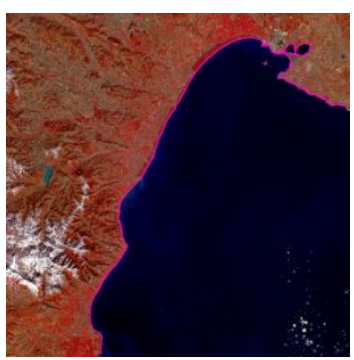

Şekil 7. Elde edilen kıyı çizgileri

Referans ikili görüntü ile sunulan yaklaşımın uygulanması sonucunda elde edilen ikili görüntünün karşılaştırılarak ortalama doğruluk sonuçları elde edilmiștir (Tablo 3). Tablo 3'teki değerlerden de görülebileceği üzere, F- Skor değeri 
\%99,79 oranına ulaşmıştır. Kıyı morfolojisinde ani ve sık değişimlerin bulunduğu test görüntülerinde (Finlandiya ve Kırım) doğruluk ölçütlerinde \%0.01 oranında düșüș olduğu görülmüștür. Ayrıca ani ve sık değișimler olmamasına rağmen dalga etkisi bulunan görüntülerde (Portekiz) doğruluk ölçütlerinde \%0.01 oranında düşüş olduğu gözlenmiştir.

Tablo 3. Test görüntüleri için ortalama doğruluk değerlendirmesi

\begin{tabular}{lllll}
\hline Doğruluk & Duyarlılık & Hassasiyet & Özgünlük & F-Skor \\
\hline 0.9917 & 0.9927 & 0.9908 & 0.9907 & 0.9917 \\
\hline
\end{tabular}

Çalışmada kullanılan eğitim ve test veri setlerine bakıldığında (115'i eğitim, 235'i test) test verisi sayısının eğitim verisinden fazla olduğu görülmektedir. Bu da kıyı çizgisi bölütlemesinde aktarımlı ögrenmede az sayıda eğitim veri seti ile yüksek doğrulukta sonuçlar üretilebileceğini göstermektedir.

\section{SONUÇLAR}

Sunulan çalışmada elde edilen sonuçlar ile literatürdeki benzer çalışmaların sonuçları karşılaştırılmıştır.

Sreekesh vd., 2019, SENTINEL-2 ve Orbview-3 uydu görüntülerinin kırmızı, yeşil, mavi ve yakın kızılötesi bantları kullanarak kıyı çizgisinin yarı otomatik olarak çıkarılmasını sağlayan bir yöntem önermiştir. Önerilen yöntem, su ve kara bölütlemesi için nesne tabanlı görüntü analizi tekniğini ve spektral öznitelik bilgilerini birleştirir. Önerilen yöntemin temel amacl, farklı spektral bantlardan elde edilen nesne bilgilerini bölütleme için kullanmaktır. Yaklaşımın performansını değerlendirmek için farklı jeomorfik özelliklere sahip kıyılar seçilmiştir ve \%95-\%99 arasında bir doğruluk başarısına ulaşmıştır. Sunulan çalışmada da farklı jeomorfolojik yedi bölge için elde edilen ortalama doğruluk \%99.17' dir.

Wieland vd., 2019 LANDSAT TM, ETM +, OLI ve SENTINEL-2 uydu görüntülerinin kırmızı, yeşil, mavi, yakın kızıötesi ve kısa dalga kızılötesi bant kombinasyonunu, sel durumlarında taşkını kalıcı sudan ayırt etmek amacıyla su sınıflarının hızlı bir şekilde bölütlenmesi için kullanmışlardır. Kara, su, buz, bulut ve gölge sınıflarını bölütlemek için evrişimli bir sinir ağı olan U-Net mimarisi kullanmışlardır ve ortalama doğruluğu \%93 olarak elde etmişlerdir.

Syrris vd., 2019 su, kara, orman, bina ve yeşil alan sınıflarını bölütlemek SENTINEL-2 uydu görüntülerin kırmızı, yeşil, mavi ve yakın kızılötesi band kombinasyonunu ve farklı CNN ( standart CNN, Fully Convolutional Network (FCN), U-net ve SegNet) modellerini kullanmışlardır. Her bir model için farklı girdi veri boyutları ( 122x122, 244x244, 366x366) kullanarak eğitim işlemi ve doğruluk değerlendirmesi yapmışlardır. En yüksek ortalama doğruluk değeri \%85 ile U-Net mimarisi ve 244x244 veri boyutu kullanılarak elde etmişlerdir. Her iki çalışma sunulan çalışma ile karşılaştırıldığında, sunulan çalışmada da U-Net mimarisi kullanılarak aktarımlı öğrenme daha yüksek bir ortalama doğruluk (\%99.17) elde edilmiștir.

Sunulan çalışmada, önceden LANDSAT-8 uydu görüntüleri ile eğitilmiş U-Net mimarisi (Erdem, vd., 2020) ve az sayıda SENTINEL-2 uydu görüntüsü veri seti kullanılarak aktarımlı öğrenme işlemi uygulanarak yüksek doğrulukta kıyı çizgisi çıkartma işleminin gerçekleştiği gösterilmiştir. SENTINEL-2 görüntülerine ait etiketli verilerin oluşturulması için OSM su poligonlarının elle düzeltilmesi ile birlikte zaman kazandıran bir yöntem olduğunu göstermiştir.

\section{TARTIŞMA}

Aralarında benzerlik bulunan görevleri ayrı ayrı değerlendirip en baştan eğitim işlemi gerçekleştirmek, yeterli sayıda eğitim veri seti hazırlamak, maliyetli ve insan gücü gerektiren bir uygulamadır. Bu çalışmada benzer görevleri ayrı ayrı eğitmek yerine daha az veri sayısı ile daha kısa sürede eğitim yapıldığı ve bilgi aktarımın mümkün olduğu aktarımlı öğrenme yöntemi kullanılmıştır. Uydu görüntüsü ile eğitilmiş bir mimarinin aktarımlı öğrenmede başka bir uydu görüntüsüyle eğitiminin kıyı çizgisi çıkarımındaki performansı araştırılmıştır. $\mathrm{Bu}$ amaç için semantik bölütleme amacıyla geliştirilen U-Net derin öğrenme mimarisi önceden eğitilmiş model olarak seçilmiştir ve aktarımlı öğrenme işlemiyle entegre edilerek az sayıdaki veri ile tekrar eğitim gerçekleștirilmiştir. Eğitim işleminin ardından 7 farklı SENTINEL-2 uydu görüntüsünden kıyı çizgisi çıkartılmıştır. Benzer iki görev arasında bilgi aktarımı yapıldığından her iki görev için de ayrı ayrı büyük veri setleri hazırlamanın önüne geçilmiştir. Böylece zaman ve insan gücünden tasarruf edilmiştir.

$\mathrm{Bu}$ çalışmada, doğruluk analizi sonuçları aktarımlı öğrenme kullanarak SENTINEL-2 uydu görüntülerinden kıyı çizgisi çıkarımınında verimli sonuçlar üretilebildiği sonucuna ulaşılmıştır. Elde edilen sonuçların kıyı yönetimi, katı madde taşınımı, kıyı çizgisi zamansal değişimi, vb. çalışmalarda kullanılabileceği değerlendirilmektedir.

\section{KAYNAKÇA}

Alesheikh, A. A., Ghorbanalı, A. \& Nouri, N. (2007). Coastline change detection using remote sensing. International Journal of Environmental Science \& Technology, 4(1), 61-66. 
Bayram, B., Erdem, F., Akpınar, B., Ince, A. K., Bozkurt, S., Reis, H. C. \& Seker, D. Z. (2017). The Efficiency of Random Forest Method for Shoreline Extraction from LANDSAT-8 and GOKTURK2 Imageries. ISPRS Annals of the Photogrammetry, Remote Sensing and Spatial Information Sciences, Volume IV-4/W4, 141145.

Choung, , Y. J. \& Jo, M. H. (2017). Comparison between a Machine-Learning-Based Method and a Water-Index-Based Method for Shoreline Mapping Using a High-Resolution Satellite Image Acquired in Hwado Island, South Korea. Journal of Sensors, Volume 2017, 8245204

Dixon, B. \& Candade, N. (2008). Multispectral landuse classification using neural networks and support vector machines: one or the other, or both. International Journal of Remote Sensing, 29(4), 1185-1206.

Erdem, F., Bayram, B., Bakirman, T., Bayrak, O.C. \& Akpinar, B. (2020). An Ensemble Deep Learning Based Shoreline Segmentation Approach (WaterNet) from Landsat 8 OLI images, Advances in Space Research, doi: https://doi.org/10.1016/j.asr.2020.10.043

Gens, R. (2010). Remote sensing of coastlines: detection, extraction and monitoring. International Journal of Remote Sensing, 31(7), 1819-1836.

Goodfellow, I., Bengio, Y., Courville, A. \& Bengio, Y. (2016). Deep Learning. MIT Press, Cambridge.

Guariglia, A., Buonamassa, A., Losurdo, A., Saladino, R., Trivigno, M. L., Zaccagnino, A. \& Colangelo, A. (2006). A multisource approach for coastline mapping \& identification of the shoreline changes. Annals of Geophysics, 49(1), 295-304.

Işıkdoğan, F., Bovik, A. C. \& Passalacqua, P. (2017). Surface Water Mapping by Deep Learning. IEEE Journal of Selected Topics in Applied Earth Observations and Remote Sensing, 10(11), 49094918.

İncekara, A. H., Seker, D. Z. \& Bayram, B. (2018). Qualifying the LIDAR-Derived Intensity Image as an Infrared Band in NDWI-Based Shoreline Extraction. IEEE Journal of Selected Topics in Applied Earth Observations and Remote Sensing, 11(12), 5053-5062.

Kalkan, K., Bayram, B., Maktav, D. \& Sunar, F. (2013). Comparison of support vector machine and object based Classification methods for coastline detection. International Archives of the Photogrammetry, Remote Sensing and Spatial Information Sciences, Volume XL-7/W2, 125-127.

Kaur, T. \& Gandhi, T. K. (2019). Deep convolutional neural networks with transfer learning for automated brain image classification. Machine Vision and Applications 31,1-16.

Lary, D. J., Alavi, A. H., Gandomi, A. H. \& Walker, A. L. (2016). Machine learning in geosciences and remote sensing. Geoscience Frontiers, 7(1), 3-10.
Li, R., Liu, W., Yang, L., Sun, S., Hu, W., Zhang, F. \& Li, W. (2018). DeepUNet: a deep fully convolutional network for pixel-level sea-land segmentation. IEEE Journal of Selected Topics in Applied Earth Observations and Remote Sensing, 11(11), 3954-3962.

Moore, L. (2000). Shoreline Mapping Techniques. Journal of Coastal Research, 16(1), 111-124. Retrieved November 18, 2020, from http://www.jstor.org/stable/4300016.

Nazerdeylami, A., Majidi, A. \& Movaghar, A. (2019). Smart Coastline Environment Management Using Deep Detection of Manmade Pollution and Hazards. 2019 5th Conference on Knowledge Based Engineering and Innovation (KBEI), Tehran, Iran, 2019, pp. 332-337

Pardo-Pascual, J. E., Almonacid-Caballer, J., Ruiz, L. A. \& Palomar-Vazquez, J. (2012). Automatic extraction of shorelines from LANDSAT TM and ETM+ multi-temporal images with subpixel precision. Remote Sensing of Environment, 123, 1-11.

Pardo-Pascual, J., Sánchez-García, E., AlmonacidCaballer, J., Palomar, J., Priego, J., FernándezSarría, A. \& Balaguer-Beser, A. (2018). Assessing the Accuracy of Automatically Extracted Shorelines on Microtidal Beaches from LANDSAT 7, LANDSAT 8 and SENTINEL-2 Imagery. Remote Sensing. 10.326. $10.3390 /$ rs10020326.

Patterson, J. \& Gibson, A. (2017). Deep Learning: A Practitioner's Approach, First Edition, O'Reilly Media, California.

Ronneberger, O., Fischer, P. \& Brox, T. (2015). U-net: Convolutional networks for biomedical image segmentation. In International Conference on Medical image computing and computerassisted intervention (pp. 234-241). Springer, Cham.

Song, S., Liu, J., Liu, Y., Feng, G., Han, H., Yao, Y. \& Du, M. (2020). Intelligent Object Recognition of Urban Water Bodies Based on Deep Learning for Multi-Source and Multi-Temporal High Spatial Resolution Remote Sensing Imagery. Sensors, 20(2), 397.

Sreekesh, S., Kaur, N. \& Sreerama Naik, S.R. (2020). An OBIA and Rule Algorithm for Coastline Extraction from High- and Medium-Resolution Multispectral Remote Sensing Images. Remote Sens Earth Syst Sci 3, 24-34

Syrris, V., Hasenohr, P., Delipetrev, B., Kotsev, A., Kempeneers, P. \& Soille, P. (2019). Evaluation of the Potential of Convolutional Neural Networks and Random Forests for Multi-Class Segmentation of SENTINEL-2 Imagery. Remote Sensing. 11. 907. 10.3390/rs11080907.

Torrey, L. \& Shavlik, J. (2009). Chapter 11 Transfer Learning.

Wieland, M., Martinis, S. \& Li, Y. (2019). Semantic segmentation of water bodies in multi-spectral satellite images for situational awareness in emergency response. ISPRS - International 
Archives of the Photogrammetry, Remote Sensing and Spatial Information Sciences. XLII2/W16. 273-277. 10.5194/isprs-archives-XLII2-W16-273-2019.

$\mathrm{Xu}, \mathrm{Y} ., \mathrm{Wu}, \mathrm{L} ., \mathrm{Xie}, \mathrm{Z}$. \& Chen, Z. (2018). Building Extraction in Very High Resolution Remote Sensing Imagery Using Deep Learning and Guided Filters. Remote Sensing, 10(1), 144-161.

Yang, L., Tian, S., Yu, L., Ye, F., Qian, J. \& Qian, Y. (2015). Deep learning for extracting water body from LANDSAT imagery. International Journal of Innovative Computing, Information and Control, 11(6), 1913-1929.

Yousef A. \& Iftekharuddin K. (2014). Shoreline extraction from the fusion of LiDAR DEM data and aerial images using mutual information and genetic algorithms. International Joint Conference on Neural Networks (IJCNN) (pp. 1007-1014). Beijing, China.

Yu S., Mou Y., Xu d., You X., Zhou L. \& Zeng, W. (2013). A New Algorithm for Shoreline Extraction from Satellite Imagery with Non-Separable Wavelet and Level Set Method. International Journal of Machine Learning and Computing, 3(1), 158163.

Yu, L., Wang, Z., Tian, S., Ye, F., Ding, J. \& Kong, J. (2017). Convolutional Neural Networks for WaterBody Extraction from Landsat Imagery. International Journal of Computational Intelligence and Applications, 16(1), 1750001.
Zhang, Y., Li, X., Zhang, J. \& Song, D. (2013). A Study on Coastline Extraction and Its Trend Based on Remote Sensing Image Data Mining. Abstract and Applied Analysis, vol. 2013, 693194. https://doi.org/10.1155/2013/693194

Zheng, G., Peng, L., Tao, G. \& Wang, C. (2011). Remote sensing analysis of Bohai Bay West Coast shoreline changes. In Proceedings IEEE International Conference on Spatial Data Mining and Geographical Knowledge Services, pp. 549552.

URL-1: http://www.remotesensinglab.yildiz.edu.tr/ [Erişim Tarihi: 30.11.2020]

URL-2:

https://SENTINEL.esa.int/web/SENTINEL/userguides/SENTINEL-2-msi/resolutions/spatial [Erişim Tarihi: 18.11.2020]

URL-3:

https://osmdata.openstreetmap.de/data/waterpolygons.html

[Erişim Tarihi: 21.11.2020]

URL-4: https://github.com/tensorflow/tensorflow [Erişim Tarihi: 21.11.2020]

URL-5: https://github.com/keras-team/keras [Erişim Tarihi: 21.11.2020] 